\title{
Identifiability with constraints
}

\author{
S. T. Glad and L. Ljung \\ Department of Electrical Engineering \\ Linköping University, S-581 83 Linköping, Sweden \\ WWW: http://www.control.isy.liu.se \\ Email: torkel@isy.liu.se, ljung@isy.liu.se
}

May 4, 1999

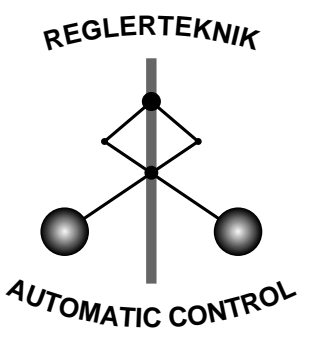

LINKÖPING

Report no.: LiTH-ISY-R-2129

Presented at NOLCOS98

Technical reports from the Automatic Control group in Linköping are available by anonymous ftp at the address ftp.control.isy.liu.se. This report is contained in the compressed postscript file 2129.ps.Z. 



\title{
Identifiability with constraints
}

\author{
S. T. Glad and L. Ljung \\ Department of Electrical Engineering, \\ Linköping University, \\ 58183 Linköping, Sweden, \\ Email: torkel@isy.liu.se, ljung@isy.liu.se \\ www.control.isy.liu.se
}

May 4, 1999

Keywords: Identifiability, differential algebra, constrained parameters, nonlinear systems.

\begin{abstract}
It is shown that identifiability of very general nonlinear models, described by polynomial implicit differential equations, inequations and inequalities, can be decided algorithmically. An algorithm based on differential algebra and real algebra can perform the calculations.
\end{abstract}

\section{Introduction}

Identifiability of parameters in mathematical models is of both intrinsic and practical interest. Surveys of work on identifiability can be found e.g. in [1] and [11]. Some algebraically related work is found in [9]. In a series of papers [5], [4], [7], [8], identifiability of very general model structures have been investigated. It was shown that systems of implicit nonlinear differential equations that are polynomial can be reduced to a standard form, using Ritt's algorithm of differential algebra. It is then possible to decide identifiability by inspection. There are several limitations to the method. One is that the method does not use the fact that in most physical models one considers only real solutions. Nor is it possible to make use of the fact that one might a priory know certain intervals in which the physical variables have to lie. Furthermore Ritt's algorithm makes use of factorizations that can be computationally very costly. In [3] an algorithm was presented by which solvability, in the sense of real solutions, of implicit systems of differential equations and inequalities could be determined. It is the purpose of the present paper to show how that algorithm can be used to test identifiability of quite general systems.

\section{Identifiability}

We will consider dynamical systems that are described by systems of implicit differential equations in some physical variables $z$, inputs $u$ and outputs $y$, 
together with a parameter vector $\theta$ to be identified. The model of the physical system is given by a set of equations

$$
f_{j}\left(u, \ldots, u^{\left(\nu_{1}\right)}, y, \ldots, y^{\left(\nu_{2}\right)}, z, \ldots, z^{\left(\nu_{3}\right)} ; \theta\right)=0, \quad j=1, \ldots, N_{f}
$$

where $f_{j}$ are polynomials with rational coefficients. It is assumed that $u, y$, and $z$ are vector valued real functions of time, with $y^{(\nu)}$ denoting the $\nu: t h$ time derivative. For technical reasons they are assumed to belong to a differential field, see e.g. [2], [10], [6]. In practise this means that we consider only analytical solutions. In addition to (1) we assume that there are constraints on the physical variables that can be expressed as inequations and inequalities

$$
\begin{array}{ll}
g_{j}\left(u, \ldots, u^{\left(\nu_{1}\right)}, y, \ldots, y^{\left(\nu_{2}\right)}, z, \ldots, z^{\left(\nu_{3}\right)} ; \theta\right) \neq 0, & \\
h_{j}\left(u, \ldots, u^{\left(\nu_{1}\right)}, y, \ldots, y^{\left(\nu_{2}\right)}, z, \ldots, z^{\left(\nu_{3}\right)} ; \theta\right)<0, & \\
& j=1, \ldots, N_{g}
\end{array}
$$

Note that we only allow strict inequalities.

In this context it is natural to define identifiability in the following way.

Definition 1 The model (1), (2), (3) is (globally) identifiable if it is impossible to find $u, y, z_{a}, z_{b}, \theta_{a}, \theta_{b}$ such that

- $u, y, z_{a}, \theta_{a}$ satisfy (1), (2), (3)

- $u, y, z_{b}, \theta_{b}$ satisfy (1), (2), (3)

- $\theta_{a} \neq \theta_{b}$

The definition means that there is no input-output pair $u, y$ which is consistent with two different values of $\theta$.

\section{Main result}

One can now show

Theorem 1 It is possible to decide algorithmically, using a finite number of additions, multiplications and symbolic differentiations, whether a system (1), (2), (3) is identifiable.

Proof: The system being non-identifiable is equivalent to solvability of the system

$$
\begin{gathered}
f_{j}\left(u, \ldots, u^{\left(\nu_{1}\right)}, y, \ldots, y^{\left(\nu_{2}\right)}, z_{a}, \ldots, z_{a}^{\left(\nu_{3}\right)} ; \theta_{a}\right)=0 \\
f_{j}\left(u, \ldots, u^{\left(\nu_{1}\right)}, y, \ldots, y^{\left(\nu_{2}\right)}, z_{b}, \ldots, z_{b}^{\left(\nu_{3}\right)} ; \theta_{b}\right)=0, \\
g_{j}\left(u, \ldots, u^{\left(\nu_{1}\right)}, y, \ldots, y^{\left(\nu_{2}\right)}, z_{a}, \ldots, z_{a}^{\left(\nu_{3}\right)} ; \theta_{a}\right) \neq 0 \\
g_{j}\left(u, \ldots, u^{\left(\nu_{1}\right)}, y, \ldots, y^{\left(\nu_{2}\right)}, z_{b}, \ldots, z_{b}^{\left(\nu_{3}\right)} ; \theta_{b}\right) \neq 0 \\
h_{j}\left(u, \ldots, u^{\left(\nu_{1}\right)}, y, \ldots, y^{\left(\nu_{2}\right)}, z_{a}, \ldots, z_{a}^{\left(\nu_{3}\right)} ; \theta_{a}\right)<0 \\
h_{j}\left(u, \ldots, u^{\left(\nu_{1}\right)}, y, \ldots, y^{\left(\nu_{2}\right)}, z_{b}, \ldots, z_{b}^{\left(\nu_{3}\right)} ; \theta_{b}\right)<0 \\
\theta_{a} \neq \theta_{b}
\end{gathered}
$$


where the indices of $f_{j}, g_{j}$ and $h_{j}$ range over the same values as in the original model. The algorithmic solvability of a system of this form was proven in [3], where also an algorithm was given.

Note 1. To reduce the identifiability problem to the solvability of (4) is conceptually the easiest way, since it is just a reformulation of the definition. When one does explicit calculations it might not be the best strategy however, since the number of equations and the number of internal variables are doubled. It might be better to compute an equivalent model first, where all internal variables are eliminated. This can be done using the algorithm of [3].

Note 2. If the system in not identifiable, the algorithm that decides identifiability will give a set of equations, inequations and inequalities characterizing an input-output pair that is consistent with different values of $\theta$. It might then be possible to impose experimental conditions in the form of additional inequations and inequalities that rule out this solution and thus restore identifiability. An example of this is shown in Example 3 below.

It is outside the scope of the paper to include the full algorithm and proof of [3]. However we will indicate the basic ideas behind the calculations that are performed.

Suppose we have the equations

$$
f_{1}\left(y, \ldots, y^{(n)}\right)=0, f_{2}\left(y, \ldots, y^{(m)}\right)=0
$$

where $n>m$ and $y$ is scalar for simplicity. By differentiating $f_{2} n-m$ times one gets an expression which is of degree one in $y^{(n)}$. Using polynomial division one obtains an expression

$$
S f_{1}=Q f_{2}^{(n-m)}+R
$$

where $R$ does not contain $y^{(n)}$. In the simplest situation $S \neq 0$ would be among the given inequations. The system $f_{1}=0, f_{2}=0$ is then equivalent to $R=0$, $f_{2}=0$. The latter system is simpler due to the removal of the highest derivative from $f_{1}$. If $S \neq 0$ is not among the given inequations, one has to consider the two systems

$$
f_{1}=0, f_{2}=0, S=0
$$

and

$$
f_{1}=0, f_{2}=0, \quad S \neq 0
$$

The latter system is equivalent to

$$
R=0, \quad f_{2}=0, S \neq 0
$$

Performing repeated calculations of this form on all the variables one shows that existence of a solution of the original system of equations is equivalent to the existence of a solution of at least one of an increasing number of successively simpler systems. Many of these will trivially lack a solution due to the presence of the equation $1=0$ or the inequation $0 \neq 0$. The others can be reduced to a standard form, called an "autoreduced set", see [3], [2], [10], [6]. It was shown in [3] that there exists a solution to this reduced set of differential equations, 
inequations and inequalities, provided one can find initial conditions consistent with the inequations and inequalities. The latter problem can be resolved using algorithmic methods from real algebra, for instance cylindrical algebraic decomposition. The whole problem is therefore algorithmically solvable (but possibly with very high complexity).

\section{Examples}

Example 1 A simple example of identifiability is the so called "Goodwin's napkin example".

$$
\ddot{y}+2 \theta \dot{y}+\theta^{2} y=0
$$

In [8] it was shown that the system can be reduced to a form that is linear in $\theta$, provided certain non-singularity conditions are met. Global identifiability then follows. Within the present framework we can formulate the identifiability problem as follows. Does there exist a solution of the system

$$
\begin{aligned}
\ddot{y}+2 \theta_{a} \dot{y}+\theta_{a}^{2} y & =0 \\
\ddot{y}+2 \theta_{b} \dot{y}+\theta_{b}^{2} y & =0 \\
\dot{\theta}_{a}=0, \quad \dot{\theta}_{b} & =0 \\
\theta_{a}-\theta_{b} \neq 0, \quad y & \neq 0
\end{aligned}
$$

The last inequation is added to exclude the trivial case $y=0$ which is obviously not identifiable. Using the algorithm given in [3], the system of equations and inequations is reduced to the system

$$
1=0, \quad \theta_{a}-\theta_{b} \neq 0, \quad y \neq 0
$$

which obviously has no solution. The system is thus globally identifiable provided $y$ is not identically zero.

Example 2 Consider a problem which is obviously not identifiable

$$
\dot{y} \ddot{y}+\theta^{2} y=0
$$

It is clear that $\theta$ is not identifiable, since its sign can be changed without affecting $y$. It is also intuitively clear that the a priory knowledge that $\theta>0$ should suffice to ensure identifiability. It is however instructive to see how this is handled algorithmically. The algorithm will reduce the system

$$
\begin{aligned}
\dot{y} \ddot{y}+\theta_{a}^{2} y & =0 \\
\dot{y} \ddot{y}+\theta_{b}^{2} y & =0 \\
\dot{\theta}_{a}=0, \quad \dot{\theta}_{b} & =0 \\
\theta_{a}-\theta_{b} & \neq 0 \\
\theta_{a}>0, \quad \theta_{b} & >0
\end{aligned}
$$

to

$$
\begin{array}{r}
\dot{\theta}_{a}=0, \theta_{a}+\theta_{b}=0, \quad \dot{y} \ddot{y}+\theta_{a}^{2} y=0 \\
\dot{y} \neq 0, \quad \theta_{a} \neq 0 \\
\theta_{a}>0, \quad-\theta_{a}>0
\end{array}
$$


(and four other systems that trivially lack solutions). The theory says that we can find a solution to this system if we can find an initial condition. The inequalities $\theta_{a}>0$ and $-\theta_{a}>0$ are however obviously incompatible, so no solution exists.

Example 3 Goodwin's Napkin example with input. Suppose we consider the model

$$
\ddot{y}+2 \theta \dot{y}+\theta^{2} y=u
$$

We then investigate the existence of solutions to the system

$$
\begin{array}{r}
\ddot{y}+2 \theta_{a} \dot{y}+\theta_{a}^{2} y=u \\
\ddot{y}+2 \theta_{b} \dot{y}+\theta_{b}^{2} y=u \\
\dot{\theta}_{a}=0, \quad \dot{\theta}_{b}=0 \\
\theta_{a}-\theta_{b} \neq 0
\end{array}
$$

In this case we do not include any condition on $y$. Running the algorithm we then get five different system of equations. Three of these lack solutions. The remaining two both have solutions. Examining the reduced set of equations one finds

$$
-u \ddot{u}+\dot{u}^{2}=0
$$

in one of them and

$$
u=0
$$

in the other one. So identifiability is only lost for these particular choices of $u$. We can verify this by adding the inequations

$$
-u \ddot{u}+\dot{u}^{2} \neq 0, \quad u \neq 0
$$

to the system (5), and as expected the algorithm shows that there are no solutions, i.e. under these conditions the system is identifiable.

\section{Conclusions}

We have shown that identifiability of a wide class of models can be decided algorithmically. The algorithm uses ideas from differential algebra and real algebra. The main advantage compared to the methods suggested in [8] is that the present algorithm does not make use of any factorization, which is an operation of high complexity. The algorithm can thus be much more efficient. However, there are still much room for algorithmic improvements.

\section{Acknowledgment}

This work was supported by the Swedish Research Council for Engineering Sciences (TFR), which is gratefully acknowledged. 


\section{References}

[1] M. J. Chappell and Keith R. Godfrey. Global identifiability of the parameters of nonlinear systems with specified inputs: A comparison of methods. Mathemtical Biosciences, 102:41-73, 1990.

[2] M. Fliess and S. T. Glad. An algebraic approach to linear and nonlinear control. In H. L. Trentelman and J. C. Willems, editors, Essays on control: Perspectives in the theory and its applications., pages 223-267. Birkhäuser, 1993.

[3] S. T. Glad. Stabilizable regions of unstable systems. Zurich, 1997. COSY Workshop on Control of Nonlinear and Uncertain Systems.

[4] S. T. Glad and L. Ljung. "Model Structure Identifiability and Persistence of Excitation". In Proc. 29th CDC, pages 3236-3240, Honululu, Hawaii, December 1990.

[5] S. T. Glad and L. Ljung. "Parametrization of nonlinear model structures as linear regressions". In Preprints, 11th IFAC World Congress, pages 67-71, August 1990.

[6] E.R. Kolchin. Differential Algebra and Algebraic Groups. Academic Press, New York, 1973.

[7] L. Ljung and S. T. Glad. Testing global identifiability for arbitrary model parameterizations. In Preprints IFAC/IFORS Symposium on System Identification and System Parameter Estimation, pages 1077-1082, Budapest, Hungary, July 1991.

[8] L. Ljung and S. T. Glad. On global identifiability of arbitrary model parameterizations. Automatica, 30(2):pp 265-276, February 1994.

[9] F. Ollivier. Generalized standard bases with applications to control. In Proc. First European Control Conf., volume 1, pages 170-176, Grenoble, France, July 1991. Hermès.

[10] J. F. Ritt. Differential Algebra. American Mathematical Society, Providence, R I, 1950.

[11] E. Walter and L. Pronzato. Qualitative and quantitative experiment design for phenomenological modelds - a survey. Automatica, 26:195-213, 1990. 